\title{
An IoT-aware AAL System to Capture Behavioral Changes of Elderly People
}

\author{
Luca Mainetti, Luigi Patrono, Andrea Secco, and Ilaria Sergi
}

\begin{abstract}
The ageing of population is a phenomenon that is affecting the majority of developed countries around the world and will soon affect developing economies too. In recent years, both industry and academia are focused on the development of several solutions aimed to guarantee a healthy and safe lifestyle to the elderly. In this context, the behavioral analysis of elderly people can help to prevent the occurrence of Mild Cognitive Impairment (MCI) and frailty problems. The innovative technologies enabling the Internet of Things (IoT) can be used in order to capture personal data for automatically recognizing changes in elderly people behavior in an unobtrusive, low-cost and low-power modality. This work aims to describe the ongoing activities within the City4Age project, funded by the Horizon 2020 Programme of the European Commission, mainly focused on the use of IoT technologies to develop an innovative AAL system able to capture personal data of elderly people in their home and city environments. The proposed architecture has been validated through a proof-of-concept focused mainly on localization issues, collection of ambient parameters, and user-environment interaction aspects.
\end{abstract}

Index Terms - Behavior Analysis, Bluetooth Low Energy, Cloud, Internet of Things, MCI, Smart Environments.

\section{INTRODUCTION}

$\mathrm{I}^{\mathrm{N}}$ $\mathrm{N}$ recent years, due a rapidly ageing society, many efforts have been made in order to develop systems able to monitor the behavior of the elderly and to support them in daily life activities. In fact, it is estimated that $50 \%$ of the population in Europe will be over 60 years old in 2040, while in the USA it is estimated that one in every six citizens will be over 65 years old in 2020 [1]. In 75-year-old people, the risk of Mild Cognitive Impairment (MCI) and frailty increases. While some physical and mental decline is part of the healthy ageing process, premature decline may be an early indicator of more severe

Manuscript received January 13, 2017; revised May, 12, 2017. Date of publication: June 1, 2017.

This work was funded by the City4Age project that has received funding from the European Union's Horizon 2020 research and innovation Programme under grant agreement No 68973. The City4Age project will activate urban communities to facilitate the role of social/health services and of families facing Mild Cognitive Impairment (MCI) and frailty of the elderly population.

Authors are with the Innovation Engineering Department, University of Salento, Via per Monteroni, 73100, Lecce, Italy (emails: \{luca.mainetti, luigi.patrono, andrea.secco, ilaria.sergi\}@unisalento.it).

Digital Object Identifier (DOI): 10.24138/jcomss.v13i2.374 conditions, such as Alzheimer's disease (AD).

The early detection of risks related to a specific health condition can help clinicians to enact appropriate interventions that can slow down the progression of the condition itself, with beneficial effects on both patients' quality of life and treatment's costs.

Ambient Assisted Living (AAL) approach is the way to guarantee better life conditions for the aged and for monitoring their health conditions by the development of innovative technologies and services. AAL technologies can also provide more safety for the elderly, offering emergency response mechanisms [2], fall detection solutions [3], and video surveillance systems [4]. Moreover, other AAL technologies were designed in order to provide support in the daily life, by monitoring the activities of daily living (ADL) [5], by generating reminders [6], as well as by allowing older adults to connect with their family and the medical staff.

Moreover, recent advancements in mobile and wearable sensors helped the vision of AAL to become a reality. All recent mobile devices are equipped with different sensors such as accelerometer, gyroscope, Global Positioning System (GPS) and so on, which can be used for detecting user mobility and motility. Furthermore, recent advances in electronic and microelectromechanical sensors (MEMS) technology promise a new era of sensor technology for health [7]. Researchers have already developed noninvasive sensors in form of patches, small holter-type devices, wearable devices, and smart garments to monitor health signals. For example, blood glucose, blood pressure, and cardiac activity can be measured through wearable sensors using techniques such as infrared or optical sensing. Some other measurements, such as Electroencephalography (EEG), still require invasive sensors such as electrodes.

Indoor and outdoor localization is another key component in AAL systems that allows to track, to monitor, and to provide fine-grained location-based services of the elderly. GPS is the most widespread and reliable technology to deal with outdoor localization issues. Nevertheless, in indoor scenarios, GPS has a limited usage due to its limited accuracy and the impact of obstacles on received signals. Therefore, a number of alternative indoor positioning systems have been proposed in the literature [8]. Among these technologies, vision techniques 
guarantee high accuracy levels. In the context of homecare, in [9] a video-based monitoring system for the elderly care is elderly independence and increase the efficiency of the homecare practices. The main disadvantage of the video technology lies in the cost, which is still too high, especially for systems with very high precision. For this reason, with the spread of mobile devices, the interest in indoor location systems using smartphones equipped with video camera [10], [11] is increased.

The infrared (IR) technology is also widely used for indoor localization, as shown in [12] and [13], though multipath effect drastically reduces the localization accuracy.

Radio Frequency Identification (RFID) is one of the most popular wireless technologies for tracing and tracking [14], [15], [16]. The main advantage of this technology is the capability to work in absence of Line of Sight ( $\mathrm{LoS}$ ) condition.

Bluetooth (BT) technology represents a valid alternative for indoor localization [17], [18]. It can guarantee a low cost since it is integrated in most of the daily used devices such as tablets and smartphones. Moreover, the spread of the emerging Bluetooth Low Energy (BLE) technology makes the BT also energy-efficient, which is a key requirement in many indoor applications. The recent rise of iBeacons by Apple has contributed to the rapid spread of this technology, used to provide information and location services [19] in a completely innovative way.

This paper aims to present an architecture that exploits the innovative technologies enabling the Internet of Things (IoT) [20] in order to capture personal data for automatically recognizing changes in elderly people behavior in an unobtrusive, low-cost and low-power manner. By using these technologies, it is possible to perform a behavioral analysis of elderly people to prevent the occurrence of MCI and frailty problems. This work is an extension of the results presented in [21]. Respect to the previous work, the system architecture was refined and enriched according to the City4Age [22] project activities. The project aims to create an innovative framework on ICT tools and services that can be deployed by European cities, in order to: enhance early detection of risk related to frailty and MCI, and provide personalized intervention that can help the elderly population to improve their daily life and also promote positive behavior changes [23]. A specific module related to the user-environment interaction was introduced. By using this module, it is possible to retrieve information about the interaction of the elderly with the surrounding environment, especially with home appliances, and detect her/his cooking habits. The proposed system has been validated through a functional approach and, for this purpose, a proof of concept has been described by supporting an elderly people in her/his daily activities. The validation has exploited one of the pilots of the City4Age project located in the city of Lecce (Italy). A further improvement reported in the current work is related to a brief comparison of the proposed system with some similar systems presented in the literature. It is worth noting that the presented work aims to meet only a part of the overall City4Age proposed. The main objectives of this system are to preserve the

project objectives, i.e. the automatic capture of personal data of the elderly people in an unobtrusive, low-cost and low power modality by using IoT technologies. Everything concerning the risk detection and the management of interventions in order to prevent the occurrence of frailty or MCI is part of the research area of the City4Age project, but it is not treated in this paper.

The paper is structured as follows. In Section II, a brief overview on the literature concerning systems to support older people and recognize their behavior is presented. Section III illustrates some details related to the City4Age project, whereas in Section IV the proposed system architecture is described. Section $\mathrm{V}$ describes the system validation from a functional point of view by focusing mainly on indoor/outdoor localization of elderly people and, in Section VI, a brief comparison among the proposed architecture and some systems presented in the literature is reported. Finally, conclusions are drawn in Section VII.

\section{RELATED WORKS}

Many solutions were proposed in the literature in order to create smart environments and applications to support elderly people. The main purpose is to provide a level of independence at home and improve elderly quality of life. In [24], a solution for tracking the daily life activities, by using mobile devices and Cloud computing services, is presented. This system can collect heterogeneous information from the sensors located in the house and share them in the Cloud. The system monitors the elderly people, their activities (by using cameras and RFID tags), and can generate reminders for scheduled activities and alerts for critical situations to care givers and family members, so reducing the health expenditures. A solution for monitoring patients with specific diseases such as diabetes using mobile devices is discussed in [25]. This system provides continuous monitoring and real time services, collecting the information from healthcare and monitoring devices located in the home environment and connected to BT mobile devices. The sensor data are transmitted to a central database for medical server evaluation and monitoring via $3 \mathrm{G}$ and Wi-Fi networks. An ad hoc application, installed on a mobile phone, allows the remote control of all their patient's health status whilst the patient can receive any notifications from the health care professionals via the application running on her/his mobile phone.

In [26], a monitoring and tracking system for people with medical problems is proposed. The solution includes a system for locomotor activity monitoring through accelerometers and Wi-Fi networks. An interactive approach involves the user, through a smart TV. The locomotor activity of the elderly people is deduced by the analysis of the Received Signal Strength Indication (RSSI) measurements, i.e. by determining through an algorithm the received signal power from different access points located in the house. Mobile accelerometers are used to analyze the movement of the user and detect steps. In this way, it is possible to obtain adequate precision at a low cost. 
Single board computers, such as Raspberry $\mathrm{Pi}$, are used to collect data coming from the different sensors wirelessly connected to obtain real-time context-aware information such as gas, temperature, fire, etc. or to get information from biomedical sensors such as, oxygen meter, blood pressure, ECG, accelerometer, etc. Moreover, Raspberry Pi can be connected to the TV in order to transmit warnings or notifications coming from the health care professionals.

Different techniques and algorithm have been discussed in the literature for human activity recognition and fall detection and, in recent years, there has been a growing interest in these activities by using data coming from accelerometers, gyroscopes and inertial sensors installed in the smartphone. By using data collected via smartphone's sensors and the surrounding environment is possible to identify the actions carried out by a human. In the literature, several data fusion and machine learning techniques have been developed and tested for this purpose. In [27] the recognition of the human activities is performed combining inertial sensors for body motion capture, a machine learning algorithm for activity prediction, and a filter of consecutive predictions for output refinement. The developed system architecture targets real-time classification with a collection of inertial sensors while addressing issues regarding the occurrence of transitions between activities and unknown activities to the learning algorithm. Authors propose two implementations of the architecture, which differ in their prediction technique as they deal with transitions either by directly learning them or by considering them as unknown activities. In [28] authors present an activity recognition and fall detection system, which used built in sensors with alarm notification service. Signal Magnitude Vector (SMV) algorithm is used to analyze the fall like events. To overcome the false alarm activation problem, system uses different threshold values to determine the daily life activities like walking, standing, and sitting, which could be wrongly detected as a fall. For assessment, a trial setup is done to acquire sensor's information of diverse positions. Finally, [29] presents an unobtrusive smartphone based fall detection system that uses a combination of information derived from machine learning classification applied in a state machine algorithm. The data from the smartphone built-in accelerometer is continuously screened when the phone is in the user's belt or pocket. Upon the detection of a fall event, the user location is tracked and SMS and email notifications are sent to a set of contacts.

Solutions based on cameras exploit complex and expensive video systems and several cameras installed in the environment. Other solutions require the installation of a complex hardware infrastructure, as in the case of RFID systems, or they need to rely on an existing infrastructure, as occurs in Wi-Fi based systems. The proposed system is able to capture behavioral changes in elderly people by exploiting unobtrusive, low-cost and low power-solutions. Finally, it is easily scalable and extensible in order to include other functionalities not currently provided.

\section{City4Age Project Description}

"City4Age - Elderly-friendly city services for active and healthy", is a research and innovation project funded by the European Commission under the Horizon 2020 Programme. The first and core objective of City4Age is to enable Ambient Assisted Cities or Age-friendly Cities. The project aims to create an innovative framework on ICT tools and services that can be deployed by European cities in order to: enhance early detection of risk related to frailty and $\mathrm{MCI}$, and provide personalized intervention that can help the elderly population to improve their daily life and also promote positive behavior changes. City4Age will activate urban communities to facilitate the role of social/health services and of families in dealing with cognitive impairments and frailty in the elderly population. The challenge is to demonstrate that cities play a pivotal role in the early detection of (MCI and frailty) risks and subsequent interventions. The core idea is that smart cities can collect data in an unobtrusive way about individual behaviors. This data can be used in two ways: (i) to identify segments of the population potentially at risk and, (ii) to closely monitor individuals already identified as specifically in need of close monitoring. In the latter case, negative changes of behaviors can be detected, prompting individualized intervention. The project involves several academia and industrial partners, coordinated by Polytechnic of Milan: Centre National De La Recherche Scientifique (FR), Polytechnic of Milan, Universidad De La Iglesia De Deusto (ES), University of Pavia (IT), Universidad Politécnica de Madrid (ES), University College London (UK), University of Salento (IT), Athens Technology Center SA (GR), Future Cities Lab LTD (UK), Geomobile GMBH (DE), MultiMed Engineers SRLS (IT), Belit DOO (SR). The City4Age project is also characterized by six pilots to test the outcomes of the research and located in six different cities: Athens, (GR), Lecce (IT), Birmingham (UK), Madrid (ES), Montpellier (FR) and Singapore.

One of the main objectives of the City4Age project is focused on the "Unobtrusive acquisition of personal data", achieved using sensors that not require direct interaction with the user. This personal data capturing system is in charge of collecting large quantities of data related to the elderly person, in order to detect behaviors that are considered relevant in the City4Age risk model for triggering possible corrective interventions. In particular, it deals with all data that can be detected from the surrounding environment through a sensing infrastructure, both in the home and in the city environment. Main types of collectable data can be grouped into the following categories:

- User motility: data related to user body activities, such as motion, rest, sleep, walking, etc.

- Indoor/Outdoor localization: data involved in the process of determining the position of the user inside an indoor place, such as user's homes or shopping malls, pharmacies, churches, etc., or data related to the position of the user in outside places, like streets, parks, etc. Sometimes it is also referred as mobility.

- Ambient parameters: data concerning the quality of living condition in indoor and outdoor environments, like temperature, humidity, luminosity, etc. 
- User-Environment interaction: data related to user interaction with the surrounding environments, especially with home appliances (TVs, HVACs, etc.) and public services (products bought in a supermarket, services utilized with public transportation, etc.).

All collected data are sent to the City4Age platform, where elderly people's behaviors and, above all, their variations, are detected. In most cases, all data are conveyed through the smartphone that formats them according to a shared data format before send them to the shared platform, acting as a gateway. In other cases, this role is played by a smart box based on a singleboard-computer.

\section{SYSTEM ARCHITECTURE}

The proposed system architecture is composed of the two main components: (i) logical component, and (ii) hardware component.

\section{A. Overall logical architecture}

Fig. 1 shows the architecture of the developed system by highlighting the logical levels and its positioning related to the City4Age platform.

The two main components of the architecture are the Local Environment Building Block (LEBB) and the Cloud Building Block (CBB). The LEBB is responsible of (i) gathering raw data from the Sensor Network (SN) independently of both specific sensor technology and communication protocols, and (ii) of eventually processing them to calculate aggregated data for the upper layers. More in detail, the LEBB, represented by the user's smartphone, is able to abstract the heterogeneity of the physical technologies, providing a common vision of data to the core logic. To do so, LEBB is equipped with appropriate software modules, which are able to communicate with the sensing technologies according to the respective standards and protocols. This feature allows to easily extend LEBB's functionality to new technologies by developing the corresponding modules. The data collected by each module are then handled by the LEBB's core logic that pre-formats them as Low-Level Elementary Actions (LEAs), including all the available information. A LEA is the finest grain atomic information used to detect behavior of elderly people. It relates to start/stop events of user basic actions and contains additional

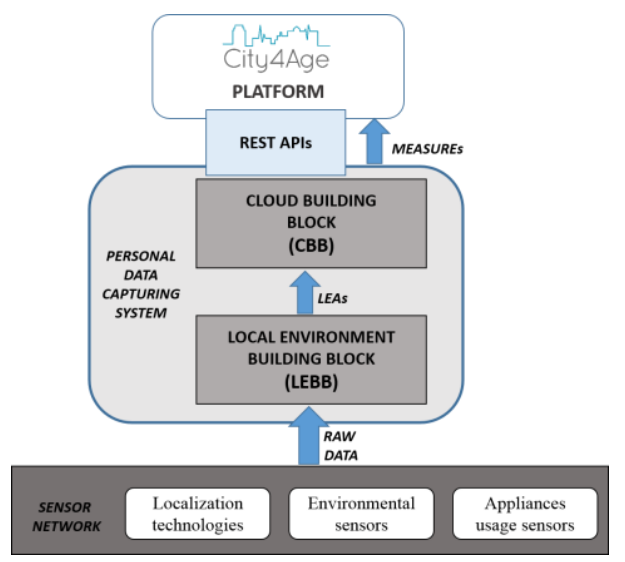

Fig. 1. Overall logical architecture. information about time and position of the action that is being taken. All this information is enveloped in the defined Common Data Format and sent to the upper layer of the City4Age Platform.

The pre-formatted LEAs are then sent through REST APIs to the $\mathrm{CBB}$, which is in charge to do some processing activities on them in order to include other available information. In some cases, in fact, the data collected by LEBB are not sufficient to calculate LEAs (e.g., if some information on user's personal profile is needed), so the LEA formatting process is completed at $\mathrm{CBB}$, which has access to a wider range of information. Furthermore, the CBB performs other computations in order to calculate MEASUREs based on the given LEAs. MEASUREs are daily indicators that make sense from a geriatric point of view in order to assess changes of behavior that are relevant for assessing MCI or frailty. Examples of MEASUREs could be: the number of calls received and done (daily), the average speed of walking (daily), the number of walks (daily), the average time spent in a given room of the home (daily), the number of visited shops (daily), etc. Based on these daily MEASUREs, further indicators can be computed in order to define a risk profile of each elderly person on a monthly base. Finally, the CBB is in charge of sending both LEAs and MEASUREs to the City4Age platform, by exploiting the uniform interface composed of the Common Data Format, defined ad hoc in the same project, and the REST APIs provided by the shared repository provided by the City4Age platform.

The SN consists of several nodes equipped with different sensors, which deliver environmental parameters, positional information, etc. More in general, all devices with sensing and connectivity capabilities, able to provide and exchange information, could be considered nodes of the network.

The designed architecture aims to provide support to the following processes:

Indoor \& outdoor localization. The use of a GPS receiver and a GPRS module, installed on a wearable device, allows the outdoor localization of the user. More in detail, the GPS module sends the position of the user, if $\mathrm{s} / \mathrm{he}$ is in outdoor environment, to the GPRS module, which in turn sends it to the LEBB. If the user is located within an indoor environment, her/his position can be derived by exploiting BLE technology. We suppose that, within each indoor environment of interest (e.g., the user's house), one or more low-cost devices equipped with BLE interface are installed, named piBeacons. The piBeacon closest to the user transmits its geographical coordinates via BLE connection to the user's wearable device, which in turn sends it to the LEBB via GPRS module, similarly to the outdoor localization. The use of the BLE technology reduces the power consumption guaranteeing a longer battery life of the wearable device. In order to ensure further energy savings, the BLE module will be disabled in the outdoor localization scenario while in the indoor localization the GPS module will be switched-off.

Ambient parameters collection. The heterogeneous devices belonging to the low level transmit different types of information to monitor data concerning the quality of living condition in indoor and outdoor environments, like 
temperature, humidity, ambient noise, rain, wind, etc. The main problem is related to the heterogeneity of the technologies supported by the network nodes. To overcome this limitation, the low-cost collector, named Smart Gateway, acts as a transparent concentrator.

\section{User-Environment interaction data collection.}

Data related to elderly interaction with home appliances (TVs, appliances, etc.), cooking habits, and care of personal hygiene are important geriatric indicators useful to detect deviations from normal behavior and then highlight risk factors related to mental fragility.

The main peculiarity of the sensing network is its unobtrusiveness. Ambient parameters are gathered through sensors included in wearable devices. Indoor localization is achieved mainly through an unobtrusive wireless system based on the BLE technology, while outdoor positioning system is based on the GPS technology. Finally, indoor user-environment interaction is achieved by exploiting ad-hoc sensors, such as humidity sensor or gas sensor to detect cooking habits or smart devices, such as smart plugs in order to detect the use of TVs or appliances. All these devices are characterized by low cost of purchasing and management. To obtain reliable information about the user's interaction with the environment, data coming from ad-hoc sensors are combined with other information, e.g. localization data. For example, in order to deduce that the elderly is watching TV, at least two information are necessary, i.e. the power consumption measured by the smart plug that is connected to the TV plug and the presence of the user in the room. Similarly, to detect cooking habits, information coming from several sensors in the kitchen are combined with user's localization data.

\section{B. Hardware components}

Fig. 2 shows the Smart Gateway (Fig. 2.a) and the prototypes used in the validation scenario in order to emulate the wearable devices (Fig. 2.b and 2.c). The main actor of the sensing middleware scenario is the Smart Gateway, a data collector realized using a low cost Single Board Computer such as a

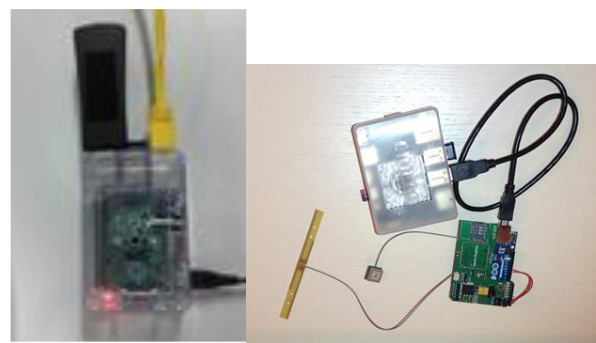

(a)

(b)

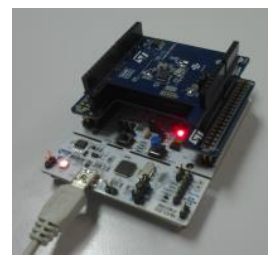

(c)

Fig. 2. Prototypes used in the validation scenario: a) Smart Gateway; b) and c) wearable devices.
Raspberry Pi [30]. The Smart Gateway performs various tasks, such as (i) data collection, (ii) data forwarding, and (iii) instructions forwarding from/to the SN. It has several input and output interfaces. In particular, the input interfaces are:

- Classic BT module: it is used to communicate with all classic BT devices;

- BLE module: it is used to communicate with all the BLE devices;

- The output interfaces used to forward the collected data are:

- Wi-Fi module: it allows the Internet access;

- Ethernet interface: installed on the Raspberry Pi board, it is used to realize a network connection via Ethernet cable.

In order to emulate the smart wristband worn by the elderly patient, two prototypal devices were realized. Fig. 2.b shows the first prototype used for the elderly indoor/outdoor localization. It was obtained by combining both Odroid [31] and Arduino [32] devices equipped with BLE, GPS and GPRS modules. This wearable device exploits the GPS technology for outdoor localization and BLE technology for indoor localization. The second prototype (Fig. 2.c) used for the detection of ambient parameters, was realized by using Nucleo Board [33] with MEMS and BLE shields [34]. In particular, MEMS shield is equipped with several sensors such as accelerometer, gyroscope, inertial modules, and environmental sensors.

The present work aims to define and test a prototype wearable device. It also may be replaced with a commercial product (e.g., the Sensor Tag [35]) that communicates values gathered from its sensors to the smartphone. By using these kind of commercial products it is possible to obtain similar results and preserve low-power and low-cost features.

\section{SYSTEM VALIDATION}

\section{A. Proof of concept}

In order to validate the proposed architecture from a functional point of view, a proof-of-concept approach has been used. In particular, the proposed scenario refers to the monitoring of an elderly in her/his home and in her/his outdoor daily activities. The elderly wears a smart device equipped with several MEMS such as accelerometers, gyroscopes, inertial modules, and environmental sensors. Another wearable device, exploiting the GPS and BLE technologies, provides indoor and outdoor localization functionalities. Furthermore, both wearable devices support data transmission capabilities. Fig. 3 shows a hypothetical indoor test scenario. A piBeacon, identified by a unique MAC address, is located in each room.

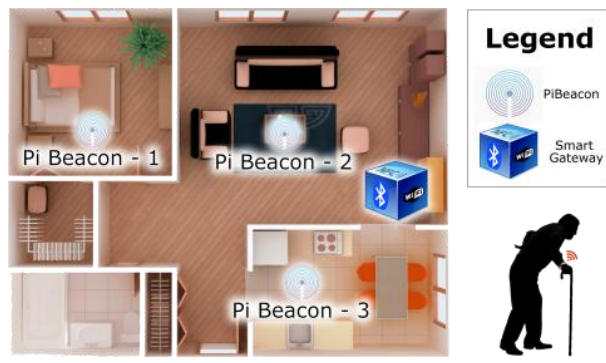

Fig. 3. Indoor test scenario. 
The wearable devices operate as nodes of the SN, delivering several data to the Smart Gateway, placed in the indoor environment. Each piBeacon communicates its coordinates via BLE. The second wearable device constantly checks the presence of piBeacons and selects the nearest by comparing the Received Signal Strength Indicator (RSSI) of detected piBeacons.

An ad hoc implemented algorithm, running on the device, is responsible for maximizing the probability of selecting the closer piBeacon. By exploiting the RSSI, the wearable device computes a proximity index $d$, using the following formula:

$$
R S S I=-(10 n \log 10 d+A)
$$

where $A$ is the received signal strength at $1 \mathrm{~m}, n$ is a signal propagation constant depending mainly on the environment, and $d$ is the distance from the sender. The piBeacon with the lowest value of $d$ represents the user's current indoor location. The indoor positional information is sent to the gateway (i.e., the elderly smartphone) through GPRS module.

When the patient leaves the indoor environment, by assuming that $\mathrm{s} /$ he wears the wristband, the GPS replaces the BLE module. The wearable device transmits the outdoor position retrieved by the GPS to the elderly smartphone via GPRS module, as in the case of the indoor localization.

Once received the elderly positions, the smartphone is responsible for formatting and sending these data to the $\mathrm{CBB}$ in order to make them available to the City4Age platform. Fig. 4 shows an example of the locomotor activity, with a detail of the room (Fig. 4.a) in which the elderly is detected and the position of the elderly in outdoor environment (Fig. 4.b), displayed by accessing to a localization Web server. In Fig. 5, a flow chart summarizes the main steps involved in the indoor/outdoor localization process.

Finally, the Smart Gateway may collect data from several environmental sensors measuring temperature, humidity,

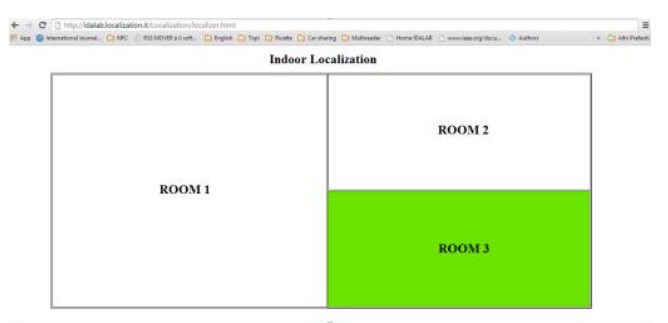

a)

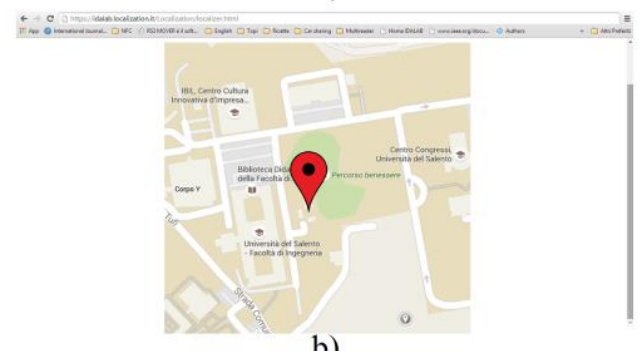

b)

Fig. 4. Screenshots of localization system: a) indoor scenario b) outdoor scenario. pressure, etc. Environmental data and positional information could be used in order to generate commands and instructions

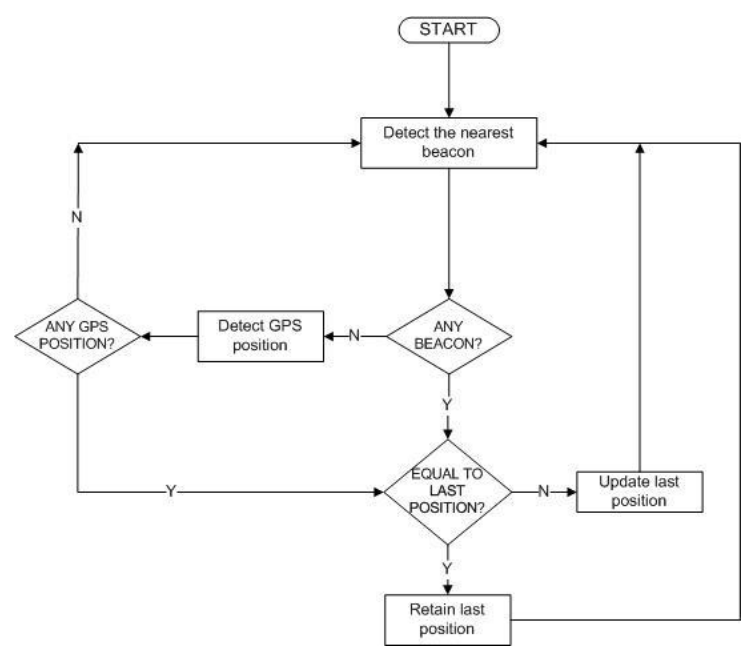

Fig. 5. Flow chart related to the indoor/outdoor positioning process.

addressed through the Smart Gateway to home devices in order to adapt the indoor environment to the needs of the elderly.

\section{B. Validation of the indoor positioning feature}

A further validation phase has been carried out in order to prove the effectiveness of the indoor positioning sub-systems.

First, a one-to-one mapping between BLE beacons and rooms has been considered, so that the room represents the granularity of the positioning algorithm. That said the correct user's localization has been tested in two different cases (Fig. 6). In the first case, referred as best case, the BLE devices of two consecutive rooms have been placed on the partition wall and not in line of sight of each other. In the second case, referred as worst case, the devices have been placed in line of sight at 5 meters from the separating door. The results shown in the Fig. 7 were obtained by positioning the wearable device (or smartphone) at three different distances from the door. The measurements allowed us to identify the Successful Localization Probability, which is the probability of correct localization of the user inside the room. The Fig. 7 shows how the results obtained are optimal in the best case and next to the ideal ones in the worst case.

\section{DISCUSSION}

In order to demonstrate the effectiveness of the proposed architecture, it was compared with similar architectures proposed in the literature. In order to carry out this comparison, some parameters were selected, i.e., the recognized activities, the typical environment in which the system works, the complexity and cost of the system. In Table I, the results of this comparison are reported. 

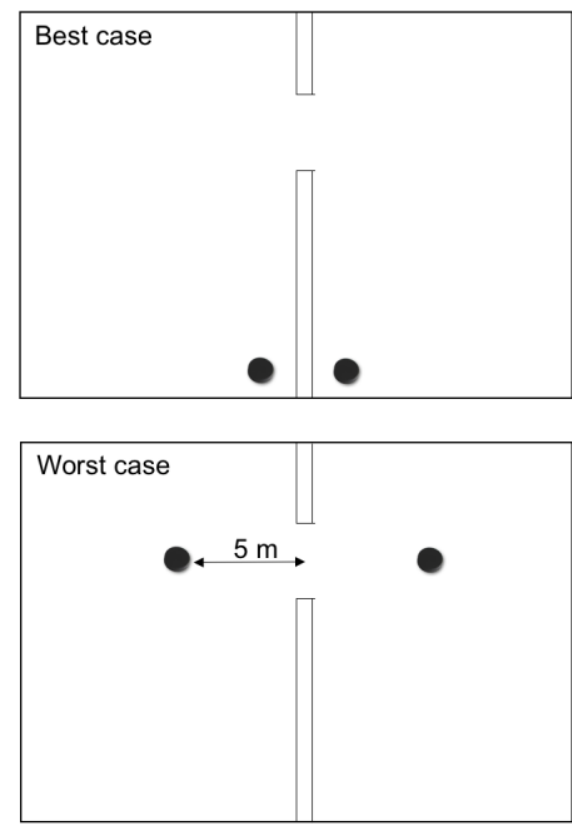

Fig. 6. Experimenting scenario of the positioning sub-system.

The main advantage of the proposed architecture is its ability to automatically recognizing behavioral changes in elderly people in an unobtrusive, low-cost and low-power modality. Furthermore, it guarantees hardware and technology

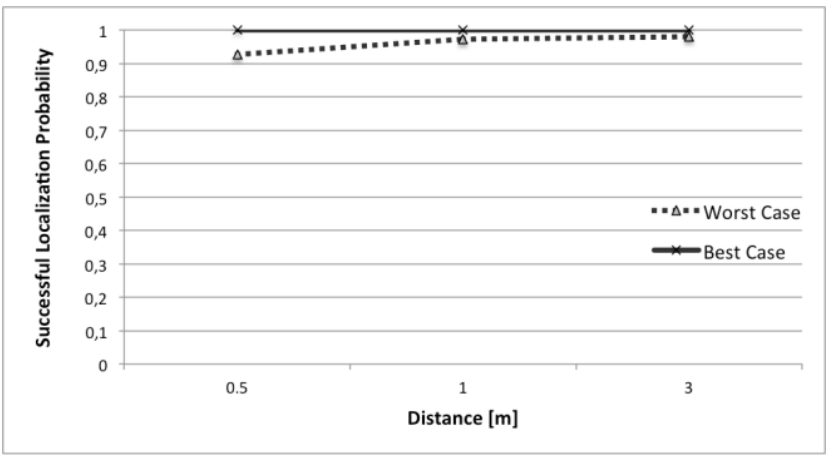

Fig. 7. Successful Localization Probability with the worst and best disposition of the BLE devices.

abstraction, since all low-level details are addressed by the adapter components included in the LEBB module, which provides as output a data object whose format is independent from any particular technology. At the same time, the proposed solution ensures high modularity and scalability, since any new technology can be added to the system simply by implementing the related adapter in LEBB. By doing so, any kind of raw data coming from heterogeneous devices can be properly handled by the system.

\section{CONCLUSION}

This work proposes an architecture aimed to monitor elderly people in their daily life. It aims to support social/health services in dealing with mild cognitive impairments and frailty
TABLE I.

COMPARISOn AMONG ElDERly MONITORING ARCHITECTURES

\begin{tabular}{|l|l|l|l|l|}
\hline$[23]$ & $\begin{array}{l}\text { Recognized } \\
\text { activities }\end{array}$ & $\begin{array}{l}\text { Typical } \\
\text { environment }\end{array}$ & Complexity & Cost \\
\hline$[\mathbf{2 4}]$ & $\begin{array}{l}\text { User- } \\
\text { Environment } \\
\text { interaction }\end{array}$ & Indoor & $\begin{array}{l}\text { Medium/ } \\
\text { Low }\end{array}$ & $\begin{array}{l}\text { Medium/ } \\
\text { High }\end{array}$ \\
\hline$[\mathbf{2 5}]$ & $\begin{array}{l}\text { Usage of } \\
\text { medical devices }\end{array}$ & Indoor & $\begin{array}{l}\text { Medium/ } \\
\text { High }\end{array}$ & Low \\
\hline$[26]$ & $\begin{array}{l}\text { Locomotor } \\
\text { activities }\end{array}$ & Indoor & Low & Low \\
\hline$[27]$ & $\begin{array}{l}\text { Locomotor } \\
\text { activities }\end{array}$ & Indoor/Outdoor & $\begin{array}{l}\text { Medium/ } \\
\text { Low }\end{array}$ & Low \\
\hline$[28]$ & Fall & Indoor/Outdoor & Medium & Low \\
\hline & Fall & Indoor/Outdoor & Medium & Low \\
\hline $\begin{array}{l}\text { Proposed } \\
\text { architecture }\end{array}$ & $\begin{array}{l}\text { Indoor/Outdoor } \\
\text { localization, } \\
\text { Ambient } \\
\text { parameters } \\
\text { collection, User- } \\
\text { Environment } \\
\text { interaction }\end{array}$ & Indoor/Outdoor & Low & Low \\
\hline
\end{tabular}

in the elderly population. The proposed system is able to guarantee three important features: (i) continuous localization in both outdoor and indoor environment, (ii) continuous monitoring of ambient parameters, and (iii) continuous monitoring of appliances usage. The system is able to transparently collect sensor data coming from heterogeneous devices and forward them to the central repository within the City4Age platform through REST APIs. From here, by applying Big Data analytics, potential risky behaviors can be identified and highlighted.

As ongoing work, we are working to realize a low cost system able to both capture the elderly cooking habits and understand if $\mathrm{s} /$ he take care of her/his personal hygiene. This system exploits smart plugs and other sensors connected to a single-board computer (e.g., a Raspeberry Pi).

\section{REFERENCES}

[1] J. M. Corchado, J. Bajo, and A. Abraham, "GerAmi: Improving Healthcare Delivery in Geriatric Residences," IEEE Intelligent Systems, vol. 23(2), 2008, pp. 19-25.

[2] Yong Lin, Xingjia Lu, Fang Fang, and Jianbo Fan, "Personal Health Care Monitoring and Emergency Response Mechanisms," First Int. Symp. on Future Information and Communication Technologies for Ubiquitous HealthCare, Jinhua , 1-3 July 2013, pp. 1-5.

[3] A. Lombardi, M. Ferri, G. Rescio, M. Grassi, and P. Malcovati, "Wearable Wireless Accelerometer with Embedded FallDetection Logic for Multi-Sensor Ambient Assisted Living Applications," IEEE Sensors, Christchurch, 25-28 Oct. 2009, pp. 1967-1970.

[4] L. Meinel, M. Findeisen, M. Heß, A. Apitzsch, and G. Hirtz, "Automated Real-Time Surveillance for Ambient Assisted Living Using an Omnidirectional Camera," IEEE Int. Conf. on Consumer Electronics (ICCE), Las Vegas, NV, 10-13 Jan. 2014, pp. 396 399.

[5] E. Kantoch, P. Augustyniak, M. Markiewicz, and D. Prusak, "Monitoring activities of daily living based on wearable wireless body sensor network," 36th Annual Int. Conf. of the IEEE Engineering in Medicine and Biology Society $(E M B C)$, Chicago, IL, 26-30 Aug. 2014, pp. 586-589. 
[6] J. A. Uribe, J. F. Duitama, and N. G. Gómez, "Personalized Message Emission in a Mobile Application for Supporting Therapeutic Adherence," 13th Int. Conf. on e-Health Networking Applications and Services (Healthcom), Columbia, MO, 13-15 June 2011, pp. 15-20.

[7] S. Vohnout, M. Engelman, and E. Enikov, "Miniature MEMSbased data recorder for prognostics and health management (PHM)," IEEE AUTOTESTCON, Orlando, FL, 13-16 Sept. 2010 , pp. 1-8.

[8] L. Mainetti, L. Patrono, and I. Sergi, "A survey on indoor positioning system," Proc. of 22nd Int. Conf. on Software, Telecommunications and Computer Networks, SoftCOM 2014, Split, 17-19 Sept. 2014, pp. 111-120.

[9] Z. Zhongna, C. Xi, C. Yu-Chia, H. Zhihai, T. X. Han, and J. M. Keller, "Video-based activity monitoring for indoor environments," IEEE Int, Symp. on Circuits and Systems, Taipei, 24-27 May 2009, pp. 1449-1452.

[10] B. Ausmeier, T. Campbell, and S. Berman, "Indoor Navigation Using a Mobile Phone," African Conf. on Software Engineering and Applied Computing (ACSEAC), Gaborone, 24-26 Sept. 2012, pp. 109-115.

[11] W. Elloumi et al., "Indoor navigation assistance with a Smartphone camera based on vanishing points," Int. Conf. on Indoor Positioning and Indoor Navigation (IPIN), Montbeliard, Fance, 28-31 Oct. 2013, pp. 1-9.

[12] D. Hauschildt and N. Kirchhof, "Advances in thermal infrared localization: Challenges and solutions," Proc. of Int. Conf. on Indoor Positioning and Indoor Navigation (IPIN), 15-17 September 2010, pp. 1-8.

[13] G. Monaci and A. Pandharipande, "Indoor user zoning and tracking in passive infrared sensing systems," Proc. of European Signal Processing Conference (EUSIPCO), Bucharest, 27-31 August 2012, pp. 1089-1093.

[14] L. Catarinucci, R. Colella, M. De Blasi, V. Mighali, L. Patrono, and L. Tarricone, "High performance RFID tags for item-level tracing systems," in 18th International Conference on Software, Telecommunications and Computer Networks, SoftCOM 2010, Split-Bol (Croatia), 2010, pp. 21-26.

[15] L. Xiaoming, P. Jing, and L. Tao, "A novel indoor localization system based on passive RFID technology," Int. Conf. on Electronic and Mechanical Engineering and Information Technology (EMEIT), Harbin, Heilongjiang, 12-14 Aug. 2011, pp. 4285-4288.

[16] L. Catarinucci, R. Colella, L. Mainetti, L. Patrono, S. Pieretti, I. Sergi, and L. Tarricone, "Smart RFID antenna system for indoor tracking and behavior analysis of small animals in colony cages," IEEE Sensors Journal, 14(4), 2014, pp. 1198-1206.

[17] W. Yapeng, Y. Xu, Z. Yutian, L. Yue, and L. Cuthbert, "Bluetooth positioning using RSSI and triangulation methods," IEEE Consumer Communications and Networking Conference (CCNC), Las Vegas, NV, 11-14 Jan. 2013, pp. 837-842.

[18] S. Alletto, R. Cucchiara, G. Del Fiore, L. Mainetti, V. Mighali, L. Patrono, and G. Serra, "An Indoor Location-aware System for an IoT-based Smart Museum, " IEEE Internet of Things Journal, vol. 3 (2), 2015, pp. 244-253. DOI: 10.1109/JIOT.2015.2506258

[19] S. Kajioka, T. Mori, T. Uchiya, I. Takumi, and H. Matsuo, "Experiment of indoor position presumption based on RSSI of Bluetooth LE beacon," IEEE 3rd Global Conf. on Consumer Electronics (GCCE), Tokyo, 7-10 Oct. 2014, pp. 337-339.

[20] L. Mainetti, V. Mighali, L. Patrono, "A software architecture enabling the web of things," IEEE Internet of Things Journal, 2 (6), 2015, pp. 445-454.
[21] L. Mainetti, L. Patrono, A. Secco, and I. Sergi, "An IoT-aware AAL system for elderly people," 1st International Multidisciplinary Conference on Computer and Energy Science, SpliTech 2016, Split (Croatia), 13 July 2016.

[22] "City4Age Project." [Online]. Available: http://www.city4ageproject.eu/. [Accessed: January 13, 2016].

[23] L. Mainetti, L. Patrono and P. Rametta, "Capturing behavioral changes of elderly people through unobtruisive sensing technologies," 2016 24th International Conference on Software, Telecommunications and Computer Networks (SoftCOM), Split, Croatia, 2016, pp. 1-3. doi: 10.1109/SOFTCOM.2016.7772126

[24] M. Fahim, I. Fatima, S. Lee, Y-K. Lee, "Daily life activity tracking application for smart homes using android smartphone", IEEE 14th International Conference on Advanced Communication Technology (ICACT), pp. 241-245, 2012.

[25] V. Villarreal, J. Fontecha, R. Hervás, J. Bravo, "Mobile and ubiquitous architecture for the medical control of chronic diseases through the use of intelligent devices: Using the architecture for patients with diabetes", Future Generation Computer System, vol. 34, pp. 161-175, 2014.

[26] G. Villarrubia, J. Bajo, J. F. de Paz, and J. M. Corchado, "Monitoring and detection platform to prevent anomalous situations in home care," Sensors, vol. 14, no. 6, pp. 9900-9921, 2014.

[27] J. Reyes-Ortiza, L. Oneto, A. Sam, A. Ghio, X. Parra, and D. Anguita, "Transition-Aware Human Activity Recognition Using Smartphones", Neurocomputing, vol. 171, 1 Jan. 2016, pp. 754767.

[28] M.B. Rasheed, N. Javaid, T. Ali Alghamdi, S. Mukhtar, U. Qasim, Z. Ali Khan, M.H.B. Raja, "Evaluation of Human Activity Recognition and Fall Detection Using Android Phone," 2015 IEEE 29th International Conference on Advanced Information Networking and Applications (AINA), 24-27 March 2015, pp. 163 -170 .

[29] B. Aguiar, T. Rocha, J. Silva, I. Sousa, "Accelerometer-based fall detection for smartphones," 2014 IEEE International Symposium on Medical Measurements and Applications (MeMeA), 11-12 June 2014.

[30] "Raspberry Pi." [Online]. Available: https://www.raspberrypi.org/. [Accessed: January 13, 2016].

[31] "Odroid." [Online]. Available: http://www.hardkernel.com/. [Accessed: January 13, 2016].

[32] "Arduino." [Online]. Available: https:// www.arduino.cc. [Accessed: January 13, 2016].

[33] "Nucleo Board (STMicroelectronics)." [Online]. Available: http://www.st.com/nucleoF401RE-pr.

[Accessed: January 13, 2016].

[34] "Bluetooth low energy expansion board (STMicroelectronics)." [Online].

Available: http://www.st.com/web/catalog/tools/FM116/SC1075/PF260517 [Accessed: January 13, 2016].

[35] "TI Sensor Tag." [Online]. Available: http://www.ti.com/ww/en/wireless_connectivity/sensortag2015/? INTC $=$ SensorTag\&HQS=sensortag [Accessed: January 13, 2016]. 


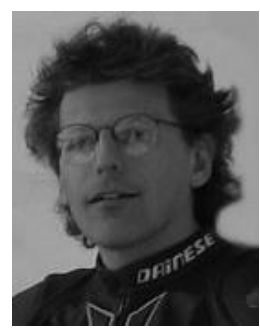

Luca Mainetti is an Associate Professor of Software Engineering and Computer Graphics at the University of Salento. His research interests include web design methodologies, notations and tools, services oriented architectures and IoT applications, and collaborative computer graphics. $\mathrm{He}$ is a scientific coordinator of the GSA Lab Graphics and Software Architectures Lab and IDA Lab - IDentification Automation Lab at the Department of Innovation Engineering, University of Salento.

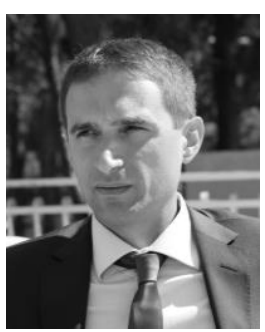

Luigi Patrono received his MS in Computer Engineering from University of Lecce, Lecce, Italy, in 1999 and $\mathrm{PhD}$ in Innovative Materials and Technologies for Satellite Networks from ISUFI-University of Lecce, Lecce, Italy, in 2003. He is an Assistant Professor of Network Design at the University of Salento, Lecce, Italy. His research interests include RFID, EPCglobal, Internet of Things, Wireless Sensor Networks, and design and performance evaluation of protocols. He is Organizer Chair of the international Symposium on RFID Technologies and Internet of Things within the IEEE SoftCOM conference. He is author of about 100 scientific papers published on international journals and conferences and four chapters of books with international diffusion.

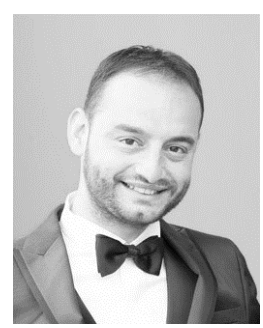

Andrea Secco received the Master's degree in Computer Engineering with honors at the University of Salento, Lecce, Italy, in 2013. His thesis concerned the tracking of small laboratory animals, based on passive UHF RFID technology. Since June 2013 he collaborates with IDA Lab - IDentification Automation Laboratory - at the Department of Innovation Engineering of the University of Salento. His activity is focused on the study of new tracking systems based on RFID technology in UHF band. He is also involved in the study of new solutions for indoor environments locating and tracking and development on new services based on NFC technology.

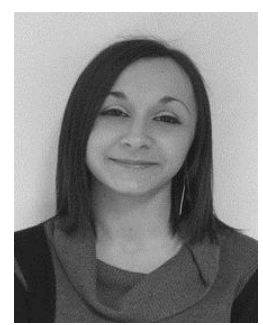

Ilaria Sergi received the Master's degree in Automation Engineering from the University of Salento, Lecce, Italy, in 2012. Her thesis focused on the tracking of small laboratory animals, based on passive UHF RFID technology. Since 2012, she has been with the IDA Laboratory and Identification Automation Laboratory, Department of Innovation Engineering, University of Salento. Her activity is focused on the study of new indoor tracking systems and homecare solutions. She has authored several papers on international journals and conferences. 